\title{
Hopf Bifurcation of a Delayed Epidemic Model with Information Variable and Limited Medical Resources
}

\author{
Caijuan Yan and Jianwen Jia \\ School of Mathematics and Computer Science, Shanxi Normal University, Linfen, Shanxi 041004, China \\ Correspondence should be addressed to Jianwen Jia; jiajw.2008@163.com
}

Received 7 January 2014; Revised 4 March 2014; Accepted 10 March 2014; Published 9 April 2014

Academic Editor: Kaifa Wang

Copyright (C) 2014 C. Yan and J. Jia. This is an open access article distributed under the Creative Commons Attribution License, which permits unrestricted use, distribution, and reproduction in any medium, provided the original work is properly cited.

\begin{abstract}
We consider SIR epidemic model in which population growth is subject to logistic growth in absence of disease. We get the condition for Hopf bifurcation of a delayed epidemic model with information variable and limited medical resources. By analyzing the corresponding characteristic equations, the local stability of an endemic equilibrium and a disease-free equilibrium is discussed. If the basic reproduction ratio $\mathscr{R}_{0}<1$, we discuss the global asymptotical stability of the disease-free equilibrium by constructing a Lyapunov functional. If $\mathscr{R}_{0}>1$, we obtain sufficient conditions under which the endemic equilibrium $E^{*}$ of system is locally asymptotically stable. And we also have discussed the stability and direction of Hopf bifurcations. Numerical simulations are carried out to explain the mathematical conclusions.
\end{abstract}

\section{Introduction}

From an epidemiological viewpoint, it is important to investigate the global dynamics of the disease transmission. In the literature, many authors have researched various epidemic models $[1,2]$, in which the stability analyses have been carried out extensively. In the recent years, based on SIR epidemic model, in order to investigate the spread of an infectious disease transmitted by a vector, Wang et al. [3] have considered the asymptotic behavior of the following delayed SIR epidemic model:

$$
\begin{aligned}
& \frac{d S(t)}{d t}=r S\left(1-\frac{S}{k}\right)-\beta S I(t-\tau), \\
& \frac{d I(t)}{d t}=\beta S I(t-\tau)-\left(\mu_{1}+\gamma\right) I, \\
& \frac{d R(t)}{d t}=\gamma I-\mu_{2} R .
\end{aligned}
$$

Since nonlinearity in the incidence rates has been observed in disease transmission dynamics, it has been suggested that the standard bilinear incidence rate will be modified into a nonlinear incidence rate by many authors [46]. In [7], incidence rate $\beta S I(t-\tau)$ in (1) was replaced by a nonlinear incidence rate of the form $\beta S G(I(t-\tau))$ with the following system:

$$
\begin{aligned}
& \frac{d S(t)}{d t}=r S\left(1-\frac{S}{k}\right)-\beta S G(I(t-\tau)), \\
& \frac{d I(t)}{d t}=\beta S G(I(t-\tau))-\left(\mu_{1}+\gamma\right) I, \\
& \frac{d R(t)}{d t}=\gamma I-\mu_{2} R .
\end{aligned}
$$

In order to control the spread of epidemic, we consider the new variable $Z$ :

$$
Z(t)=\int_{-\infty}^{t} S \frac{1}{T} \exp \left(-\frac{1}{T}(t-\tau)\right) d \tau,
$$

called information variable which summarizes information about the current state of the disease, that is, depending on current values of state variables, and also summarizes information about past values of state variables. Many authors have used this variable in their models (see, e.g., [8-10]).

In this paper, we consider the information variable $Z(t)$, nonlinear incidence rate of the form $\beta S G(I(t-\tau))$, and limited 
medical resources $h(I)=b I /(\omega+I)$. The model can be described by the following system of equations:

$$
\begin{aligned}
\frac{d S(t)}{d t} & =r S\left(1-\frac{S}{k}\right)-\beta S G(I(t-\tau)) \\
\frac{d I(t)}{d t} & =\beta Z G(I(t-\tau))-\left(\mu_{1}+\gamma+\varepsilon\right) I-\frac{b I}{\omega+I} \\
Z(t) & =\int_{-\infty}^{t} S \frac{1}{T} \exp \left(-\frac{1}{T}(t-\tau)\right) d \tau, \\
\frac{d R(t)}{d t} & =\gamma I-\mu_{2} R+\frac{b I}{\omega+I}
\end{aligned}
$$

where $S(t), I(t), R(t)>0$ and $S(t), I(t), R(t)$ denote the numbers of susceptible, infective, and recovered individuals at time $t$, respectively. $r$ is the intrinsic growth rate of susceptibles, $k$ is the carrying capacity of susceptibles, $\alpha$ is the saturation factor that measures the inhibitory effect, $\beta$ is the transmission or contact rate, $\mu_{1}, \mu_{2}$ are the natural death rate of the infective and recovered individuals, $\gamma$ is the natural recovery rate, $\varepsilon$ is the disease-related mortality, $b \geq 0$ is the maximal medical resources supplied per unit time, and $\omega>0$ is half-saturation constant. $r, b, \mu_{1}, \mu_{2}, \gamma, \alpha, \beta, k, \omega$ are all positive.

We further assume that the function $G$ is continuous on $[0,+\infty)$ and continuously differentiable on $(0,+\infty)$ satisfying the following hypotheses:

(1) $G(I)$ is strictly monotone increasing on $[0,+\infty)$ with $G(0)=0$;

(2) $I / G(I)$ is monotone increasing on $(0,+\infty)$ with $\lim _{I \rightarrow 0+}(I / G(I))=1$.

The organization of this paper is as follows. In Section 2, we explore the existence of disease-free equilibria point and the unique existence of the endemic equilibrium point. In Section 3, we analyze the stability of the disease-free equilibria. In Section 4, we obtain sufficient conditions under which the endemic equilibrium $E^{*}$ of system is locally asymptotically stable. In Section 5, we also have discussed the stability and direction of Hopf bifurcations. A numerical analysis and a simple discussion are given to conclude this paper in Section 6.

\section{The Existence of Equilibria}

The nonlinear integrodifferential system (4) can be transformed into the following set of nonlinear ordinary differential questions:

$$
\begin{aligned}
& \frac{d S(t)}{d t}=r S\left(1-\frac{S}{k}\right)-\beta S G(I(t-\tau)), \\
& \frac{d I(t)}{d t}=\beta Z G(I(t-\tau))-\left(\mu_{1}+\gamma+\varepsilon\right) I-\frac{b I}{\omega+I}, \\
& \frac{d Z(t)}{d t}=\frac{1}{T}(S-Z) \\
& \frac{d R(t)}{d t}=\gamma I-\mu_{2} R+\frac{b I}{\omega+I} .
\end{aligned}
$$

Since the dynamical behavior of the last equation of the system (5), that is, the dynamics of $R$, depends only the dynamics of $I$, we do not consider that equation in our discussion. Here we will study the following nonlinear ordination differential equations:

$$
\begin{aligned}
& \frac{d S(t)}{d t}=r S\left(1-\frac{S}{k}\right)-\beta S G(I(t-\tau)), \\
& \frac{d I(t)}{d t}=\beta Z G(I(t-\tau))-\left(\mu_{1}+\gamma+\varepsilon\right) I-\frac{b I}{\omega+I}, \\
& \frac{d Z(t)}{d t}=\frac{1}{T}(S-Z) .
\end{aligned}
$$

For simplicity, we nondimensionalize system (6) by defining

$$
\begin{aligned}
\widetilde{S}(\tilde{t}) & =\frac{S(t)}{k}, \quad \widetilde{I}(\tilde{t})=\frac{I(t)}{k}, \quad \widetilde{Z}(\tilde{t})=\frac{Z(t)}{k}, \\
\widetilde{t} & =\beta k t, \quad \widetilde{T}=\beta k T, \quad \widetilde{\omega}=\frac{\omega}{k}, \quad \widetilde{r}=\frac{r}{\beta k}, \\
\widetilde{G}(\widetilde{I}(\widetilde{t})) & =\frac{G(I(t))}{k}, \quad \widetilde{\mu}_{1}=\frac{\mu_{1}}{\beta k}, \quad \widetilde{\mu}_{2}=\frac{\mu_{2}}{\beta k}, \\
\widetilde{\gamma} & =\frac{\gamma}{\beta k}, \quad \widetilde{\varepsilon}=\frac{\varepsilon}{\beta k}, \quad \tilde{b}=\frac{b}{\beta k^{2}} .
\end{aligned}
$$

We note that $\widetilde{G}$ also satisfies the hypotheses (1) and (2). Dropping the $\sim$ for convenience of readers, system (6) can be written in the following form:

$$
\begin{aligned}
& \frac{d S(t)}{d t}=r S(1-S)-S G(I(t-\tau)) \\
& \frac{d I(t)}{d t}=Z G(I(t-\tau))-\left(\mu_{1}+\gamma+\varepsilon\right) I-\frac{b I}{\omega+I}, \\
& \frac{d Z(t)}{d t}=\frac{1}{T}(S-Z) .
\end{aligned}
$$

The basic reproduction is $\mathscr{R}_{0}=1 /\left(\mu_{1}+\gamma+\varepsilon+b / \omega\right)$.

Theorem 1. (1) The system (8) has a trivial equilibrium $E_{0}=$ $(0,0,0)$ and the disease-free equilibrium $E_{1}=(1,0,1)$.

(2) If $\mathscr{R}_{0}>1$, the system (8) has one endemic equilibrium $E^{*}=\left(S^{*}, I^{*}, Z^{*}\right)$ except the disease-free equilibria $E_{0}$ and $E_{1}$.

Proof. (1) Let $I=0$; we have $S=Z=0$, or $S=Z=1$; it is not easy to find that the system has a trivial equilibrium and the disease-free equilibria $E_{0}=(0,0,0)$ and $E_{1}=(1,0,1)$.

(2) If $\mathscr{R}_{0}>1$, from the third question of (8), we have $Z^{*}=S^{*}$; from the second question of (8), we have

$$
S^{*}=Z^{*}=\frac{\left(\mu_{1}+\gamma+\varepsilon+b /\left(\omega+I^{*}\right)\right) I^{*}}{G\left(I^{*}\right)} .
$$

Then substituting them into the first question of (8) yields

$$
r\left[1-\frac{\left(\mu_{1}+\gamma+\varepsilon+b /\left(\omega+I^{*}\right)\right) I^{*}}{G\left(I^{*}\right)}\right]-G\left(I^{*}\right)=0 .
$$


Let $F(I)=r\left[1-\left(\mu_{1}+\gamma+\varepsilon+b /\left(\omega+I^{*}\right)\right) I / G(I)\right]-G(I)$. By hypothesis (2), we obtain

$$
\begin{aligned}
\lim _{I \rightarrow+0} F(I) & =r\left[1-\left(\mu_{1}+\gamma+\varepsilon+\frac{b}{\omega+I^{*}}\right)\right] \\
& >r\left[1-\left(\mu_{1}+\gamma+\varepsilon+\frac{b}{\omega}\right)\right]=r\left(1-\frac{1}{R_{0}}\right)>0 .
\end{aligned}
$$

Since $F(I)$ is strictly monotone decreasing function on $(0,+\infty)$, it suffices to show that $F(I)<0$ holds for $I$ sufficiently large. From (1), $G(I)$ is either unbounded above or bounded above on $[0,+\infty)$.

First, we suppose that $G(I)$ is unbounded above. Then there exists an $I_{1}>0$ such that $G\left(I_{1}\right)=r$, from which we have $F(I)<0$ for all $I \geq I_{1}$. Second we suppose that
$G(I)$ is bounded above. Then, from (2), $I / G(I)$ is unbounded above on $[0,+\infty)$; that is, there exists an $I_{2}>0$ such that $\mu_{1}+\gamma+\varepsilon+b /\left(\omega+I_{2}\right)=G\left(I_{2}\right) / I_{2}$. This yields $F(I)<0$ for all $I>I_{2}$. Therefore, for the both cases, there exists a unique endemic $I^{*}>0$ such that $F\left(I^{*}\right)=0$. By the second and third equations of (8), there exists a unique endemic equilibrium $E^{*}$ of system (8) if $\mathscr{R}_{0}>1$.

Second, we assume $\mathscr{R}_{0} \leq 1$; then it is obvious that system (8) has no equilibria. Hence the proof is complete.

\section{The Stability Analysis of Disease-Free Equilibrium Point}

In this section, we will examine the local stability of the equilibria by analyzing the eigenvalues of the Jacobian matrices of (8) at the equilibria and using Routh-Hurwitz criterion.

Let $\bar{E}=(\bar{S}, \bar{I}, \bar{R})$ be the arbitrarily equilibrium point of system (8); then the Jacobian matrix of (8) at $\bar{E}$ is

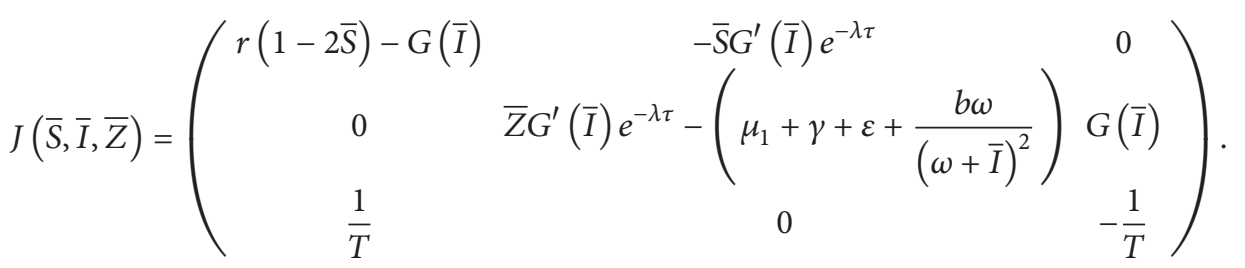

Then the characteristic equation of the system (8) at equilibrium $\bar{E}$ is

$$
\begin{aligned}
& |\lambda E-J(\bar{E})| \\
& =\left(\lambda+\frac{1}{T}\right)[\lambda+G(\bar{I})-r(1-2 \bar{S})] \\
& \quad \times\left[\lambda+\mu_{1}+\gamma+\varepsilon+\frac{b \omega}{(\omega+\bar{I})^{2}}\right] \\
& \quad-\left[\left(\lambda+\frac{1}{T}\right)(\lambda+G(\bar{I})-r(1-2 \bar{S}))-\frac{1}{T} G(\bar{I})\right] \\
& \quad \times \bar{Z} G^{\prime}(\bar{I}) e^{-\lambda \tau}=0 .
\end{aligned}
$$

Theorem 2. The trivial equilibrium $E_{0}$ of system (8) is always unstable.

Proof. The characteristic equation (13) at $E_{0}=(0,0,0)$ becomes as follows:

$$
\left(\lambda+\frac{1}{T}\right)(\lambda-r)\left(\lambda+\mu_{1}+\gamma+\varepsilon+\frac{b}{\omega}\right)=0 .
$$

Since (14) has a positive $\operatorname{root} \lambda=r>0, E_{0}$ is unstable.

Theorem 3. The disease-free equilibrium $E_{1}$ of system (8) is locally asymptotically stable if $\mathscr{R}_{0}<1$ and it is unstable if $\mathscr{R}_{0}>1$.
Proof. For $E_{1}=(1,0,1)$, the characteristic equation (13) at $E_{1}$ becomes as follows:

$$
\left(\lambda+\frac{1}{T}\right)(\lambda+r)\left[\lambda+\left(\mu_{1}+\gamma+\varepsilon+\frac{b}{\omega}\right)-e^{-\lambda \tau}\right]=0 .
$$

It is clear that both $\lambda=-1 / T$ and $\lambda=-r$ are all the negative root of (15). Then the other root of (15) is determined as the following equation:

$$
\lambda+\left(\mu_{1}+\gamma+\varepsilon+\frac{b}{\omega}\right)-e^{-\lambda \tau}=0 .
$$

For the case $\mathscr{R}_{0}<1$, we suppose on the contrary that $E_{1}$ is not locally asymptotically stable; that is, $\operatorname{Re} \tilde{\lambda}>0$. Then, there exists a root $\lambda=\tilde{\lambda}$, such that $\operatorname{Re} \tilde{\lambda} \geq 0$. However, from (16), we obtain

$$
\begin{aligned}
\operatorname{Re} \tilde{\lambda} & =\left(\mu_{1}+\gamma+\varepsilon+\frac{b}{\omega}\right)\left(\mathscr{R}_{0} e^{-\operatorname{Re} \tilde{\lambda} \tau} \cos (\operatorname{Im} \tilde{\lambda} \tau)-1\right) \\
& \leq\left(\mu_{1}+\gamma+\varepsilon+\frac{b}{\omega}\right)\left(\mathscr{R}_{0}-1\right)<0,
\end{aligned}
$$

which is a contradiction. Hence, if $\mathscr{R}_{0}<1$, the disease-free equilibrium $E_{1}$ of system (8) is locally asymptotically stable.

Now, we put

$$
P(\lambda)=\lambda+\left(\mu_{1}+\gamma+\varepsilon+\frac{b}{\omega}\right)-e^{-\lambda \tau}=0 .
$$

For the case $\mathscr{R}_{0}>1$, we have $P(0)=\mu_{1}+\gamma+\varepsilon+b / \omega-1<0$ and $\lim _{\lambda \rightarrow+\infty} P(\lambda)=+\infty$; then $P(\lambda)=0$ has at least one positive root. Hence, $E_{1}$ is unstable if and only if $\mathscr{R}_{0}>1$. The proof is complete. 


\section{The Stability Analysis of the Endemic Equilibrium Point}

Theorem 4. If $\tau=0, \mathscr{R}_{0}>1$, and $r-G^{\prime}\left(I^{*}\right)>$ $r T G\left(I^{*}\right)$, then the positive equilibrium $E^{*}$ of system (8) is locally asymptotically stable.

Proof. The characteristic equation of (13) at $E^{*}$ becomes as follows:

$$
\begin{aligned}
(\lambda+ & \left.\frac{1}{T}\right)\left(\lambda+r S^{*}\right)\left[\lambda+\mu_{1}+\gamma+\varepsilon+\frac{b \omega}{\left(\omega+I^{*}\right)^{2}}\right] \\
& -\left[\left(\lambda+\frac{1}{T}\right)\left(\lambda+r S^{*}\right)-\frac{1}{T} G\left(I^{*}\right)\right] Z^{*} G^{\prime}\left(I^{*}\right) e^{-\lambda \tau}=0 .
\end{aligned}
$$

The above equation can be rewritten as

$$
P(\lambda)+Q(\lambda) e^{-\lambda \tau}=0
$$

where $P(\lambda)=\lambda^{3}+b_{1} \lambda^{2}+b_{2} \lambda+b_{3}, Q(\lambda)=b_{4} \lambda^{2}+b_{5} \lambda+b_{6}$, and

$$
\begin{gathered}
b_{1}=\frac{1}{T}+r S^{*}+\mu_{1}+\gamma+\varepsilon+\frac{b \omega}{\left(\omega+I^{*}\right)^{2}}, \\
b_{2}=\frac{1}{T} r S^{*}+\frac{1}{T}\left(\mu_{1}+\gamma+\varepsilon+\frac{b \omega}{\left(\omega+I^{*}\right)^{2}}\right) \\
+r S^{*}\left(\mu_{1}+\gamma+\varepsilon+\frac{b \omega}{\left(\omega+I^{*}\right)^{2}}\right), \\
b_{3}=\frac{1}{T} r S^{*}\left(\mu_{1}+\gamma+\varepsilon+\frac{b \omega}{\left(\omega+I^{*}\right)^{2}}\right), \\
b_{5}=-S_{4}^{*} G^{\prime}\left(I^{*}\right)\left[\frac{1}{T}+r S^{*}\right], \\
b_{6}=-\frac{1}{T} S^{*} G^{\prime}\left(I^{*}\right)\left(r S^{*}-G\left(I^{*}\right)\right) .
\end{gathered}
$$

Let $C=\mu_{1}+\gamma+\varepsilon+b \omega /\left(\omega+I^{*}\right)^{2}-S^{*} G^{\prime}\left(I^{*}\right)$.

Then if $\tau=0$, (20) becomes $P(\lambda)+Q(\lambda)=0$; that is,

$$
\lambda^{3}+a_{1} \lambda^{2}+a_{2} \lambda+a_{3}=0
$$

where

$$
\begin{aligned}
& a_{1}=b_{1}+b_{4}=\frac{1}{T}+r S^{*}+C, \\
& a_{2}=b_{2}+b_{5}=\frac{1}{T}\left(r S^{*}+C\right)+r S^{*} C, \\
& a_{3}=b_{3}+b_{6}=\frac{1}{T}\left[r S^{*} C+S^{*} G\left(I^{*}\right) G^{\prime}\left(I^{*}\right)\right] .
\end{aligned}
$$

Let $r-G^{\prime}\left(I^{*}\right)>r T G\left(I^{*}\right)$; we have $a_{1}>0, a_{3}>0$, and

$$
\begin{aligned}
a_{1} a_{2}-a_{3}= & \left(\frac{1}{T}+r S^{*}+C\right)\left[\frac{1}{T}\left(r S^{*}+C\right)+r S^{*} C\right] \\
& -\frac{1}{T}\left[r S^{*} C+S^{*} G\left(I^{*}\right) G^{\prime}\left(I^{*}\right)\right]
\end{aligned}
$$

$$
\begin{aligned}
= & \frac{1}{T}\left(r S^{*}+C\right)\left(\frac{1}{T}+r S^{*}+C\right) \\
& +\left(r S^{*}+C\right) r S^{*} C-\frac{1}{T} S^{*} G\left(I^{*}\right) G^{\prime}\left(I^{*}\right) \\
> & \frac{1}{T}\left(r S^{*}+C\right)\left(\frac{1}{T}+r S^{*}+C\right) \\
& +\left(r S^{*}+C\right) r S^{*} C-\frac{1}{T} r S^{*} G\left(I^{*}\right) \\
= & \frac{1}{T^{2}}\left(r S^{*}+C\right) \\
& +\frac{1}{T}\left(r S^{*}+C\right)\left(r S^{*}+C+r T S^{*} C\right) \\
& -\frac{1}{T} r S^{*} G\left(I^{*}\right) \\
= & \frac{1}{T^{2}}\left(\mu_{1}+\gamma+\varepsilon+\frac{b \omega}{\left(\omega+I^{*}\right)^{2}}\right) \\
& +\frac{1}{T}\left(r S^{*}+C\right)\left(r S^{*}+C+r T S^{*} C\right) \\
& +\frac{1}{T^{2}} S^{*}\left(r-G^{\prime}\left(I^{*}\right)\right)-\frac{1}{T} r S^{*} G\left(I^{*}\right)>0 .
\end{aligned}
$$

By using the Routh-Hurwitz theorem, $\lambda$ has negative real part for $\tau=0$. So the positive equilibrium $E^{*}$ is locally asymptotically stable.

In the following, we investigate the existence of purely imaginary roots $\lambda=i \omega(\omega>0)$ to (19). Equation (19) takes the form of a third-degree exponential polynomial in $\lambda$, with all the coefficients of $P$ and $Q$ depending on $\tau$. Beretta and Kuang [11] established a geometrical criterion which gives the existence of purely imaginary root of a characteristic equation with delay dependent coefficients.

Now we let $\lambda=i \omega(\omega>0)$ be a root of (20) from which we have that

$$
\begin{aligned}
P(\lambda)+ & Q(\lambda) e^{-\lambda \tau} \\
= & -i \omega^{3}-b_{1} \omega^{2}+b_{2} \omega i+b_{3} \\
& +\left(-b_{4} \omega^{2}+b_{5} \omega i+b_{6}\right)(\cos (\omega \tau)-i \sin (\omega \tau)) \\
= & -b_{1} \omega^{2}+b_{3} \\
& -\left[b_{4} \omega^{2} \cos (\omega \tau)-b_{5} \omega \sin (\omega \tau)-b_{6} \cos (\omega \tau)\right] \\
& +i\left[-\omega^{3}+b_{2} \omega+b_{4} \omega^{2} \sin (\omega \tau)\right. \\
& \left.+b_{5} \omega \cos (\omega \tau)-b_{6} \sin (\omega \tau)\right]=0 .
\end{aligned}
$$

Hence, we have that

$$
\begin{aligned}
-b_{1} \omega^{2}+b_{3} & =b_{4} \omega^{2} \cos (\omega \tau)-b_{5} \omega \sin (\omega \tau)-b_{6} \cos (\omega \tau) \\
& =\left(b_{4} \omega^{2}-b_{6}\right) \cos (\omega \tau)-b_{5} \omega \sin (\omega \tau),
\end{aligned}
$$




$$
\begin{aligned}
\omega^{3}-b_{2} \omega & =b_{4} \omega^{2} \sin (\omega \tau)+b_{5} \omega \cos (\omega \tau)-b_{6} \sin (\omega \tau) \\
& =\left(b_{4} \omega^{2}-b_{6}\right) \sin \omega \tau+b_{5} \omega \cos (\omega \tau) .
\end{aligned}
$$

From (26), it follows that

$$
\begin{aligned}
& \cos (\omega \tau)=\frac{b_{5} \omega\left(\omega^{3}-b_{2} \omega\right)+\left(b_{3}-b_{1} \omega^{2}\right)\left(b_{4} \omega^{2}-b_{6}\right)}{\left(b_{4} \omega^{2}-b_{6}\right)^{2}+\left(b_{5} \omega\right)^{2}}, \\
& \sin (\omega \tau)=\frac{\left(\omega^{3}-b_{2} \omega\right)\left(b_{4} \omega^{2}-b_{6}\right)-b_{5} \omega\left(b_{3}-b_{1} \omega^{2}\right)}{\left(b_{4} \omega^{2}-b_{6}\right)^{2}+\left(b_{5} \omega\right)^{2}} .
\end{aligned}
$$

By the definitions of $P(\lambda), Q(\lambda)$ is as in (20), and applying the property (1), (27a) and (27b) can be written as

$$
\sin (\omega \tau)=\operatorname{Im} \frac{P(i \omega)}{Q(i \omega)}, \quad \cos (\omega \tau)=-\operatorname{Re} \frac{P(i \omega)}{Q(i \omega)},
$$

which yields $|P(i \omega)|^{2}=|Q(i \omega)|^{2}$. of

Assume that $D \in R_{0^{+}}$is the set where $\omega \tau$ is a positive root

$$
F(\omega)=|P(i \omega)|^{2}-|Q(i \omega)|^{2}
$$

From

$$
\begin{aligned}
& |P(i \omega)|^{2} \\
& =\left|-i \omega^{3}-b_{1} \omega^{2}+b_{2} \omega i+b_{3}\right|^{2} \\
& \quad=\left|\left(b_{3}-b_{1} \omega^{2}\right)+\left(b_{2} \omega-\omega^{3}\right)\right|^{2} \\
& =\omega^{6}+\left(b_{1}^{2}-2 b_{2}\right) \omega^{4}+\left(b_{2}^{2}-2 b_{1} b_{3}\right) \omega^{2}+b_{3}^{2}, \\
& |Q(i \omega)|^{2} \\
& =\left|\left(-b_{4} \omega^{2}+b_{5} \omega i+b_{6}\right)(\cos (\omega \tau)-\sin (\omega \tau))\right|^{2} \\
& =\left(b_{6}-b_{4} \omega^{2}\right)^{2}+\left(b_{5} \omega\right)^{2} \\
& =b_{4}^{2} \omega^{4}+\left(b_{5}^{2}-2 b_{4} b_{6}\right) \omega^{2}+b_{6}^{2},
\end{aligned}
$$

we have $F(\omega)=\omega^{6}+a_{1} \omega^{4}+a_{2} \omega^{2}+a_{3}$, where $a_{1}=b_{1}^{2}-2 b_{2}-b_{4}^{2}$, $a_{2}=b_{2}^{2}-2 b_{1} b_{3}-b_{5}^{2}+2 b_{4} b_{6}, a_{3}=b_{3}^{2}-b_{6}^{2}$, and, for $\tau \in D, \omega \tau$ is not defined. Then, for all $\tau$ in $D, \omega \tau$ satisfied $F(\omega)=0$.

Let $\omega^{2}=h$; then we have that

$$
F(h)=h^{3}+a_{1} h^{2}+a_{2} h+a_{3}=0 .
$$

Assume that $F(\omega)$ has only one positive real root; we denote by $h^{+}$this positive real root. Thus, (29) has only one positive real root $\omega=\sqrt{h^{+}}$. And the critical values of $\tau$ and $\omega(\tau)$ are impossible to solve explicitly, so we will use the procedure described in Beretta and Kuang [11] and Song et al. [12]. According to this procedure, we define $\theta(\tau) \in$ $[0,2 \pi)$ such that $\sin \theta(\tau)$ and $\cos \theta(\tau)$ are given by the righthand sides of (27a) and (27b), respectively, with $\theta(\tau)$ given by (19).
And the relation between the argument $\theta$ and $\omega(\tau)$ in (28) for $\tau>0$ must be

$$
\omega(\tau)=\theta+2 n \pi, \quad n=0,1,2, \ldots
$$

Hence we can define the maps $\tau_{n}: D \rightarrow R_{+0}$ given by

$$
\tau_{n}=\frac{\theta(\tau)+2 n \pi}{\omega(\tau)}, \quad \tau_{n}>0, n=1,2, \ldots
$$

where a positive root $\omega(\tau)$ of (31) exists in $D$. Let us introduce the functions

$$
\begin{array}{r}
S_{n}(\tau): D \longrightarrow R, \quad S_{n}(\tau)=\tau-\frac{\theta(\tau)+2 n \pi}{\omega(\tau)}, \\
n=0,1,2, \ldots,
\end{array}
$$

which are continuous and differentiable in $\tau$. Thus, we give the following theorem which is due to Beretta and Kuang [11].

Theorem 5. Assume that $\omega(\tau)$ is a positive root of (19) defined for $\tau \in D, D \subseteq R_{+0}$, and, at some $\tau^{*} \in D, S_{n}\left(\tau^{*}\right)=0$ for some $n \in N_{0}$. Then a pair of simple conjugate pure imaginary roots $\lambda= \pm i \omega$ exists at $\tau=\tau^{*}$ which crosses the imaginary axis from left to right if

$$
\delta\left(\tau^{*}\right)=\operatorname{sign}\left\{F_{\omega}^{\prime}\left(\omega \tau^{*}, \tau^{*}\right)\right\} \operatorname{sign}\left\{\left.\frac{d S_{n}(\tau)}{d \tau}\right|_{\tau=\tau^{*}}\right\} .
$$

Applying Theorems 2 and 3 and the Hopf bifurcation theorem for functional differential equation [13], we can conjecture the existence of a Hopf bifurcation as stated in Theorem 6.

Theorem 6 (a conjecture). For system (2), there exists $\tau^{*} \in D$, such that the equilibrium $E^{*}$ is asymptotically stable for $0 \leq$ $\tau<\tau^{*}$, and it becomes unstable for $\tau$ staying in some tight neighborhood of $\tau^{*}$, with a Hopf bifurcation occurring when $\tau=\tau^{*}$.

\section{Stability and Direction of Hopf Bifurcations}

In this section, we will study the direction of the Hopf bifurcation and stability of bifurcating periodic solutions by using the normal theory and center manifold theorem due to Hassard et al. [14]. Letting $u_{1}=S-S^{*}, u_{2}=I-I^{*}, u_{3}=Z-Z^{*}$, $\tilde{u}_{i}(t)=u_{i}(\tau t)(i=1,2,3), \tau=\nu+\tau^{*}$, and dropping the bars for simplification of notations, system (8) becomes functional differential equations in $C=C\left([-1,0], \mathbb{R}^{3}\right)$ as

$$
\begin{aligned}
u_{1}^{\prime}(t)=\left(\tau^{*}+v\right) & \\
\times & {\left[r\left(1-2 S^{*}\right) u_{1}(t)-G\left(I^{*}\right) u_{1}(t)\right.} \\
& \left.-S^{*} G^{\prime}\left(I^{*}\right) u_{2}(t-1)-G^{\prime}\left(I^{*}\right) u_{1}(t) u_{2}(t-1)\right], \\
u_{2}^{\prime}(t)= & \left(\tau^{*}+v\right) \\
& \times\left[G^{\prime}\left(I^{*}\right) u_{3}(t) u_{2}(t-1)+G\left(I^{*}\right) u_{3}(t)\right.
\end{aligned}
$$




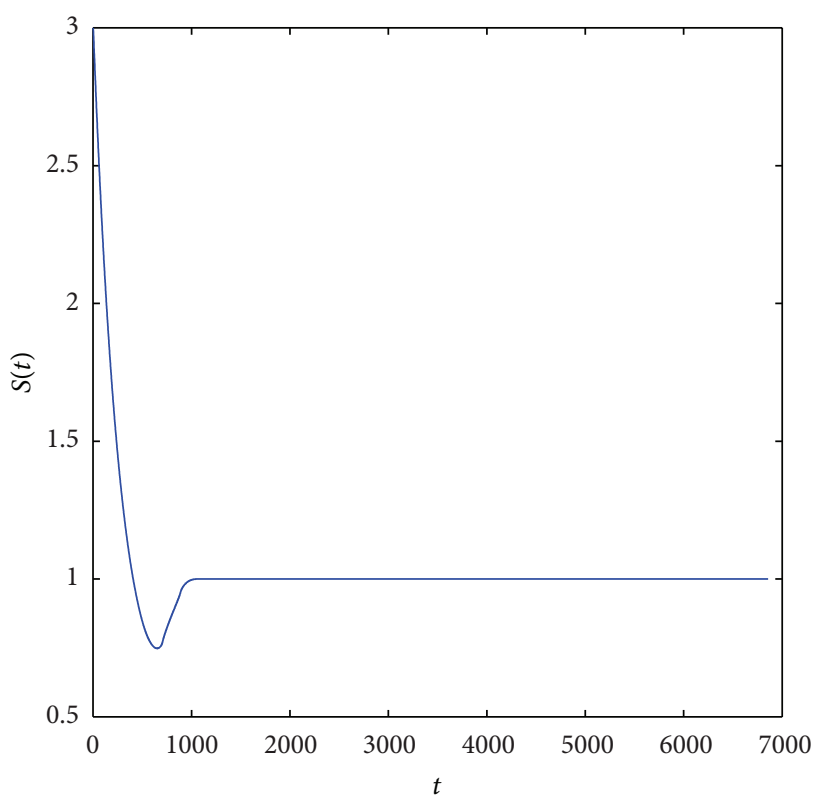

(a) Time series of $S(t)$ of the system

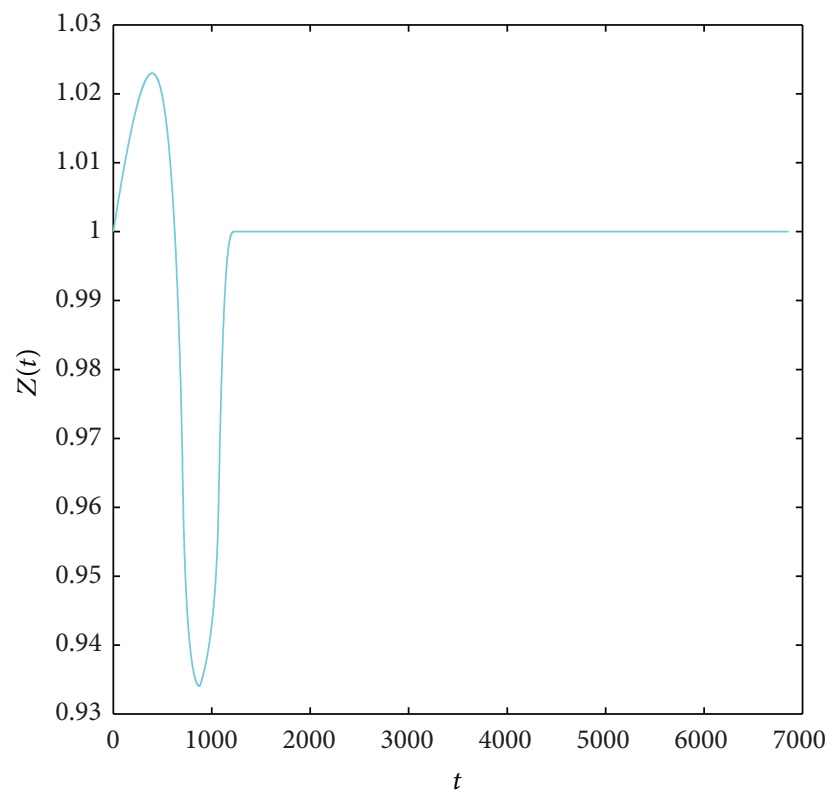

(c) Time series of $Z(t)$ of the system

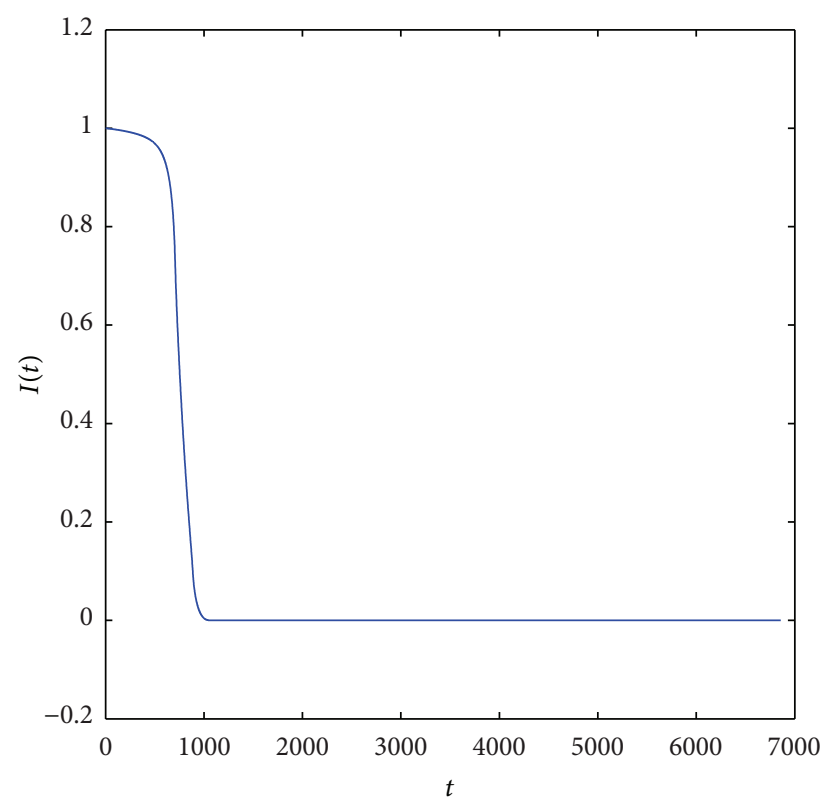

(b) Time series of $I(t)$ of the system

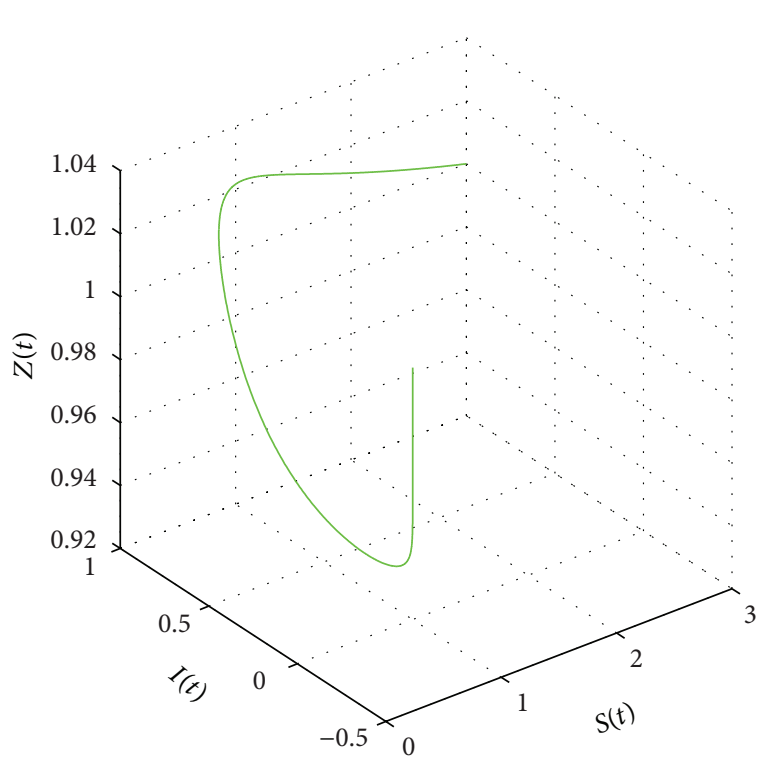

(d)

FIGURE 1: (a)-(d) showed that the equilibrium $E_{1}$ of system (8) with initial condition $S(0)=3 ; I(0)=1 ; Z(0)=1 ; R_{0}=0.3745<1 ;$ and $T=4$ is locally asymptotically stable.

$$
\begin{aligned}
& +Z^{*} G^{\prime}\left(I^{*}\right) u_{2}(t-1)-\left(\mu_{1}+\gamma+\varepsilon\right) u_{2}(t) \\
& \left.-\frac{b \omega}{\left(\omega+I^{*}\right)^{2}} u_{2}(t)+\frac{b \omega}{\left(\omega+I^{*}\right)^{3}} u_{2}^{2}(t)-\cdots\right], \\
& u_{3}^{\prime}(t)=\frac{1}{T}\left(\tau^{*}+\nu\right)\left(u_{1}(t)-u_{3}(t)\right) .
\end{aligned}
$$

Then system (36) is equivalent to

$$
u^{\prime}(t)=L_{\nu} u(t)+f(\nu, u(t))
$$

where $u(t)=\left(u_{1}(t), u_{2}(t), u_{3}(t)\right)^{T} \in \mathbb{R}^{3}$ and $L_{v}: C \rightarrow \mathbb{R}^{3}$, $f: \mathbb{R} \times C \rightarrow \mathbb{R}^{3}$ are given, respectively, by

$$
\begin{aligned}
& L_{\nu}(\phi) \\
& =\left(\tau^{*}+\nu\right)
\end{aligned}
$$

$$
\times\left(\begin{array}{ccc}
r\left(1-2 S^{*}\right)-G\left(I^{*}\right) & 0 & 0 \\
0 & -\left(\mu_{1}+\gamma+\varepsilon\right)-\frac{b \omega}{\left(\omega+I^{*}\right)^{2}} & G\left(I^{*}\right) \\
\frac{1}{T} & 0 & -\frac{1}{T}
\end{array}\right)
$$




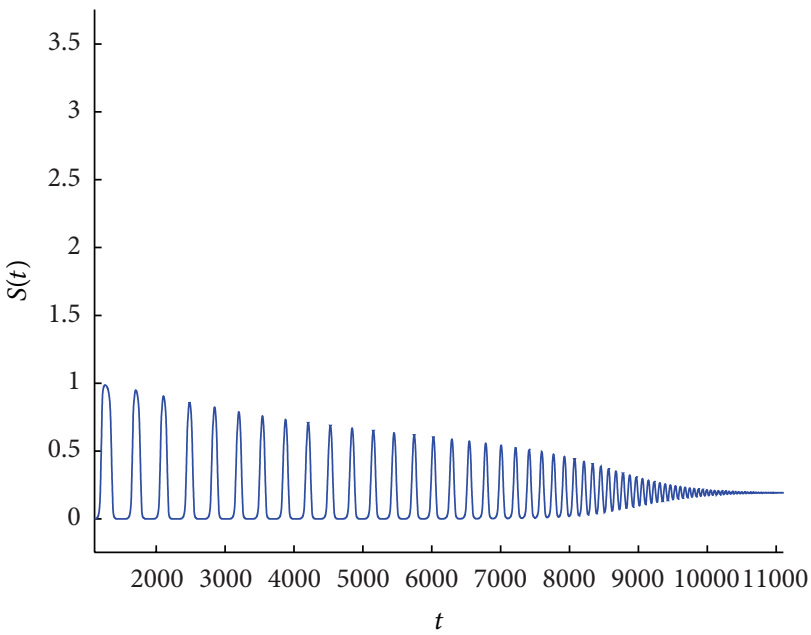

(a) Time series of $S(t)$ of the system

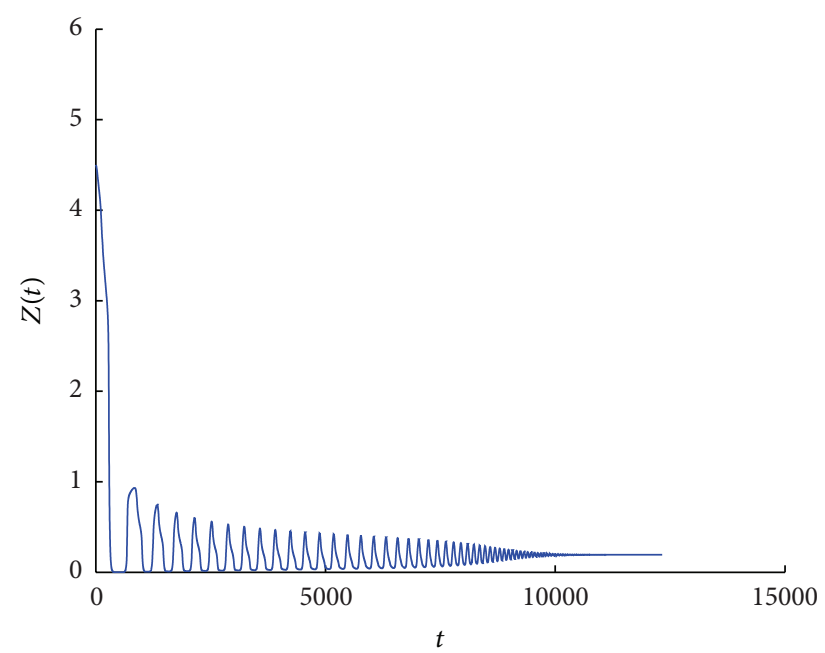

(c) Time series of $Z(t)$ of the system

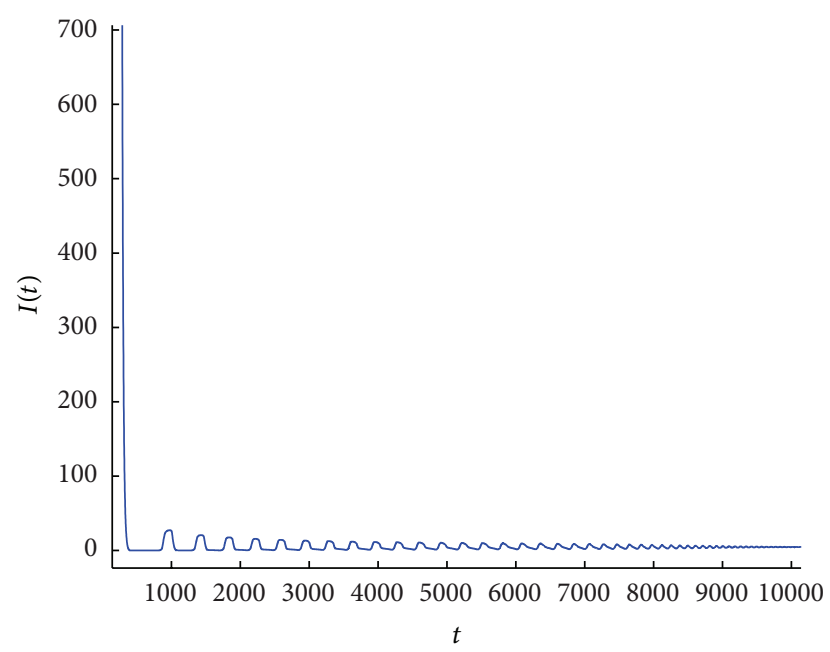

(b) Time series of $I(t)$ of the system

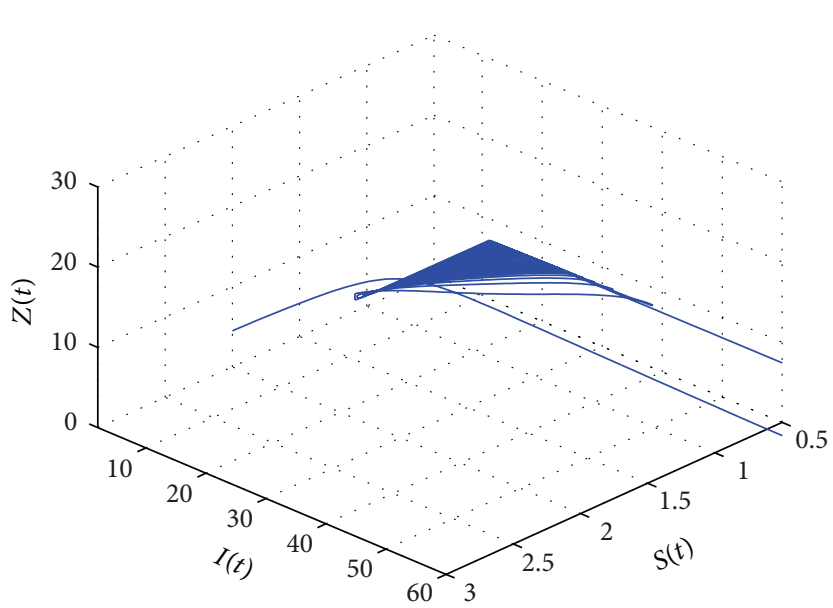

(d)

FIGURE 2: (a)-(d) showed that equilibrium $E^{*}$ of system (8) with initial condition $S(0)=2 ; I(0)=2 ; Z(0)=4.5 ; R_{0}=3.0303>1 ;$ and $\tau=1.56<\tau^{*}$ is locally asymptotically stable; that is, the trajectory converges to the positive equilibrium at $\tau=1.56$.

$$
\begin{aligned}
& \quad \times\left(\begin{array}{l}
\phi_{1}(0) \\
\phi_{2}(0) \\
\phi_{3}(0)
\end{array}\right)+\left(\tau^{*}+v\right)\left(\begin{array}{ccc}
0 & -S^{*} G^{\prime}\left(I^{*}\right) & 0 \\
0 & S^{*} G^{\prime}\left(I^{*}\right) & 0 \\
0 & 0 & 0
\end{array}\right)\left(\begin{array}{l}
\phi_{1}(-1) \\
\phi_{2}(-1) \\
\phi_{3}(-1)
\end{array}\right) \\
& f(\nu, \phi) \\
& =\left(\tau^{*}+\nu\right) \\
& \quad \times\left\{\left(\begin{array}{c}
-G^{\prime}\left(I^{*}\right) \phi_{1}(0) \phi_{2}(-1) \\
G^{\prime}\left(I^{*}\right) \phi_{3}(0) \phi_{2}(-1) \\
0
\end{array}\right)+\left(\begin{array}{c}
0 \\
\frac{b \omega}{\left(\omega+I^{*}\right)^{3}} \phi_{2}^{2}(0) \\
0
\end{array}\right)+\cdots\right\} .
\end{aligned}
$$

By the Riesz representation theorem, there exists a function $\eta(\theta, \nu)$ of bounded variation for $\theta \in[-1,0]$, such that

$$
L_{\nu}(\phi)=\int_{-1}^{0} d \eta(\theta, \nu) \phi(\theta), \quad \text { for } \theta \in C .
$$

In fact, we can choose

$$
\begin{aligned}
& \eta(\theta, v) \\
& =\left(\tau^{*}+\nu\right) \\
& \quad \times\left(\begin{array}{rrr}
r\left(1-2 S^{*}\right)-G\left(I^{*}\right) & 0 & 0 \\
0 & -\left(\mu_{1}+\gamma+\varepsilon\right)-\frac{b \omega}{\left(\omega+I^{*}\right)^{2}} & G\left(I^{*}\right) \\
\frac{1}{T} & 0 & -\frac{1}{T}
\end{array}\right) \\
& \quad \times \delta(\theta)-\left(\tau^{*}+\nu\right)\left(\begin{array}{ccc}
0 & -S^{*} G^{\prime}\left(I^{*}\right) & 0 \\
0 & S^{*} G^{\prime}\left(I^{*}\right) & 0 \\
0 & 0 & 0
\end{array}\right) \delta(\theta+1),
\end{aligned}
$$

where $\delta$ denote the Dirac delta function. For $\phi \in C([-1,0]$, $\mathbb{R}^{3}$ ), define 


$$
\begin{aligned}
A(\nu) \phi & = \begin{cases}\frac{d \phi(\theta)}{d \theta}, & \theta \in[-1,0), \\
\int_{-1}^{0} d \eta(\theta, \nu) \phi(\theta), & \theta=0,\end{cases} \\
R(\nu)(\phi) & = \begin{cases}0, & \theta \in[-1,0), \\
f(\nu, \phi), & \theta=0 .\end{cases}
\end{aligned}
$$

Then system (37) is equivalent to

$$
\dot{u}(t)=A(\nu) u_{t}+R(\nu) u_{t},
$$

where $u_{t}=u(t+\theta)$ for $\theta \in[-1,0]$.

For $\psi \in C\left([0,1],\left(\mathbb{R}^{3}\right)^{*}\right)$, define

$$
A^{*} \psi(s)= \begin{cases}-\frac{d \psi(s)}{d s}, & s \in(0,1], \\ \int_{-1}^{0} \psi(-t) d \eta(t, 0), & s=0,\end{cases}
$$

and a bilinear inner product

$$
\begin{aligned}
& \langle\psi(s), \phi(\theta)\rangle \\
& =\bar{\psi}(0) \phi(0)-\int_{-1}^{0} \int_{\sigma=0}^{\theta} \bar{\psi}(\sigma-\theta) d \eta(\theta) \phi(\sigma) d \sigma,
\end{aligned}
$$

where $\eta(\theta)=\eta(\theta, 0)$. Then $A(0)$ and $A^{*}$ are adjoint operators. By the discussion in (20), we know that $\pm i \omega^{*} \tau^{*}$ are eigenvalues of $A(0)$. Hence, they are also eigenvalues of $A^{*}$. We first need to compute the eigenvectors of $A(0)$ and $A^{*}$ corresponding to $i \omega^{*} \tau^{*}$ and $-i \omega^{*} \tau^{*}$, respectively.

Suppose that $q(\theta)=\left(1, q_{1}, q_{2}\right)^{T} e^{i \omega^{*} \tau^{*} \theta}$ is the eigenvectors of $A(0)$ corresponding to $i \omega^{*} \tau^{*}$; then $A(0) q(\theta)=i \omega^{*} \tau^{*} q(\theta)$. Then, from the definition of $A(0)$ and (38), (40), (41), and $q(-1)=q(0) e^{-i \omega^{*} \tau^{*}}$, we have

$$
\begin{gathered}
\left(\begin{array}{ccc}
r\left(1-2 S^{*}\right)-G\left(I^{*}\right) & -S^{*} G^{\prime}\left(I^{*}\right) & 0 \\
0 & S^{*} G^{\prime}\left(I^{*}\right)-\left(\mu_{1}+\gamma+\varepsilon\right)-\frac{b \omega}{\left(\omega+I^{*}\right)^{2}} & G\left(I^{*}\right) \\
\frac{1}{T} & 0 & -\frac{1}{T}
\end{array}\right) \\
\times\left(\begin{array}{c}
1 \\
q_{1}(0) \\
q_{2}(0)
\end{array}\right)=i \omega^{*}\left(\begin{array}{c}
1 \\
q_{1}(0) \\
q_{2}(0)
\end{array}\right) .
\end{gathered}
$$

We obtain

$$
q_{1}=\frac{r-2 r S^{*}-G\left(I^{*}\right)-i \omega^{*}}{S^{*} G^{\prime}\left(I^{*}\right)}, \quad q_{2}=\frac{1}{1+T i \omega^{*}} .
$$

Similarly, we can obtain the eigenvector $q^{*}(s)=$ $D\left(1, q_{1}^{*}, q_{2}^{*}\right)^{T} e^{i \omega^{*} \tau^{*}}$ of $A^{*}$ corresponding to $-i \omega^{*} \tau^{*}$, where

$$
\begin{gathered}
q_{1}^{*}=\frac{S^{*} G^{\prime}\left(I^{*}\right)}{S^{*} G^{\prime}\left(I^{*}\right)-\left(\mu_{1}+\gamma+\varepsilon\right)-b \omega /\left(\omega+I^{*}\right)^{2}+i \omega^{*}}, \\
q_{2}^{*}=T\left[-i \omega-r\left(1-2 S^{*}\right)+G\left(I^{*}\right)\right] .
\end{gathered}
$$

In order to assure that $\left\langle q^{*}(s), q(\theta)\right\rangle=1,\left\langle q^{*}(s), \bar{q}(\theta)\right\rangle=0$, we need to determine the value of $D$. By (45), we have

$$
\begin{aligned}
& \left\langle q^{*}(s), q(\theta)\right\rangle \\
& =\bar{D}\left(1, \bar{q}_{1}^{*}, \bar{q}_{2}^{*}\right)\left(1, q_{1}, q_{2}\right)^{T} \\
& -\int_{-1}^{0} \int_{\sigma=0}^{\theta} \bar{D}\left(1, \bar{q}_{1}^{*}, \bar{q}_{2}^{*}\right) e^{-i \omega^{*} \tau^{*}(\sigma-\theta)} d \eta \\
& \times(\theta)\left(1, q_{1}, q_{2}\right)^{T} e^{i \omega^{*} \tau^{*} \sigma} d \sigma \\
& =\bar{D}\left\{1+q_{1} \bar{q}_{1}^{*}+q_{2} \bar{q}_{2}^{*}\right. \\
& \left.-\int_{-1}^{0}\left(1, \bar{q}_{1}^{*}, \bar{q}_{2}^{*}\right) \theta e^{i \omega^{*} \tau^{*} \theta} d \eta(\theta)\left(1, q_{1}, q_{2}\right)^{T}\right\} \\
& =\bar{D}\left\{1+q_{1} \bar{q}_{1}^{*}+q_{2} \bar{q}_{2}^{*}+\tau^{*} q_{1} S^{*} G^{\prime}\left(I^{*}\right)\left(-1+\bar{q}_{1}^{*}\right) e^{-i \omega^{*} \tau^{*}}\right\} .
\end{aligned}
$$

Therefore, we can choose $D$ as

$$
D=\frac{1}{1+\bar{q}_{1} q_{1}^{*}+\bar{q}_{2} q_{2}^{*}+\tau^{*} \bar{q}_{1} S^{*} G^{\prime}\left(I^{*}\right)\left(-1+q_{1}^{*}\right) e^{-i \omega^{*} \tau^{*}}} .
$$

We use the way of [14] and similarly way of [3]; we obtain that the coefficients are

$$
\begin{gathered}
g_{20}=2 \tau^{*} \bar{D}\left(\bar{q}_{1}^{*}-1\right) G^{\prime}\left(I^{*}\right) q_{1}+2 \tau^{*} \bar{D} \bar{q}_{1}^{*} \frac{b \omega}{\left(\omega+I^{*}\right)^{3}} q_{1}^{2}, \\
g_{11}=2 \tau^{*} \bar{D}\left(\bar{q}_{1}^{*}-1\right) G^{\prime}\left(I^{*}\right) \operatorname{Re}\left\{q_{1}\right\} \\
+2 \tau^{*} \bar{D} \bar{q}_{1}^{*} \frac{b \omega}{\left(\omega+I^{*}\right)^{3}}\left|q_{1}\right|^{2} ; \\
g_{02}=2 \tau^{*} \bar{D}\left(\bar{q}_{1}^{*}-1\right) G^{\prime}\left(I^{*}\right) \bar{q}_{1}+2 \tau^{*} \bar{D} \bar{q}_{1}^{*} \frac{b \omega}{\left(\omega+I^{*}\right)^{3}} \bar{q}_{1}^{2} ; \\
g_{21}=\tau^{*} \bar{D}\left(\bar{q}_{1}^{*}-1\right) G^{\prime}\left(I^{*}\right) \\
+\left[W_{20}^{(1)}(0) \bar{q}_{1}+2 q_{1} W_{11}^{(1)}(0)+W_{20}^{(2)}(0)+2 W_{11}^{(2)}(0)\right] \\
+2 \tau^{*} \bar{D} \bar{q}_{1}^{*} \frac{b \omega}{\left(\omega+I^{*}\right)^{3}}\left[\bar{q}_{1} W_{20}^{(2)}(0)+2 q_{1} W_{11}^{(2)}(0)\right],
\end{gathered}
$$

where

$$
\begin{aligned}
W_{20}(\theta)= & \frac{i g_{20}}{\omega^{*} \tau^{*}} q(0) e^{i \omega^{*} \tau^{*} \theta} \\
& +\frac{i \bar{g}_{02}}{3 \omega^{*} \tau^{*}} \bar{q}(0) e^{-i \omega^{*} \tau^{*} \theta} \\
& +\left[W_{20}(0)+\frac{g_{20}}{i \omega^{*} \tau^{*}} q(0)+\frac{\bar{g}_{02}}{3 i \omega^{*} \tau^{*}} \bar{q}(0)\right] e^{2 i \omega^{*} \tau^{*} \theta}
\end{aligned}
$$




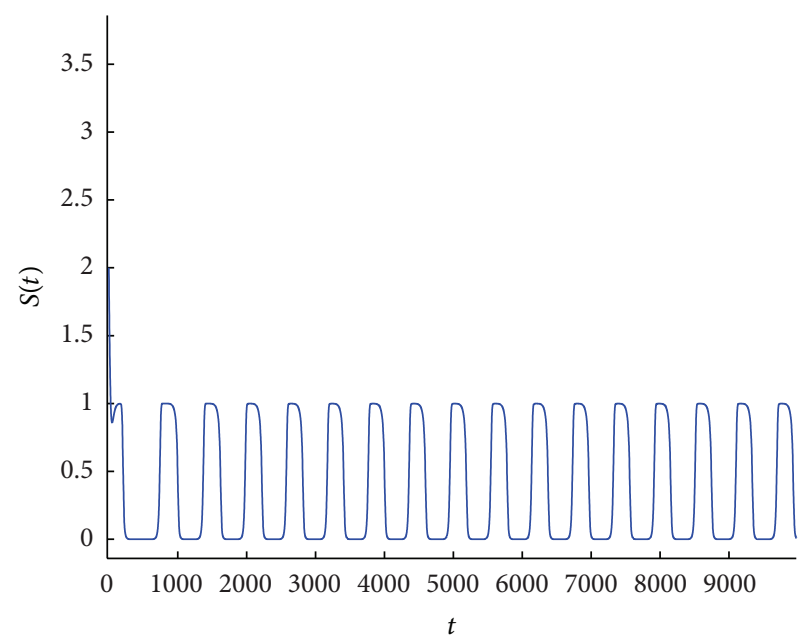

(a) Time series of $S(t)$ of the system

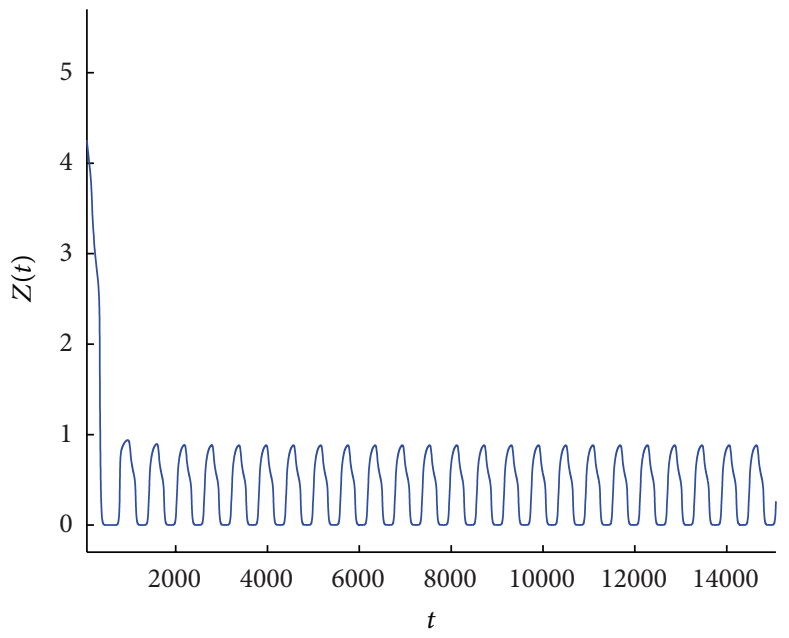

(c) Time series of $Z(t)$ of the system

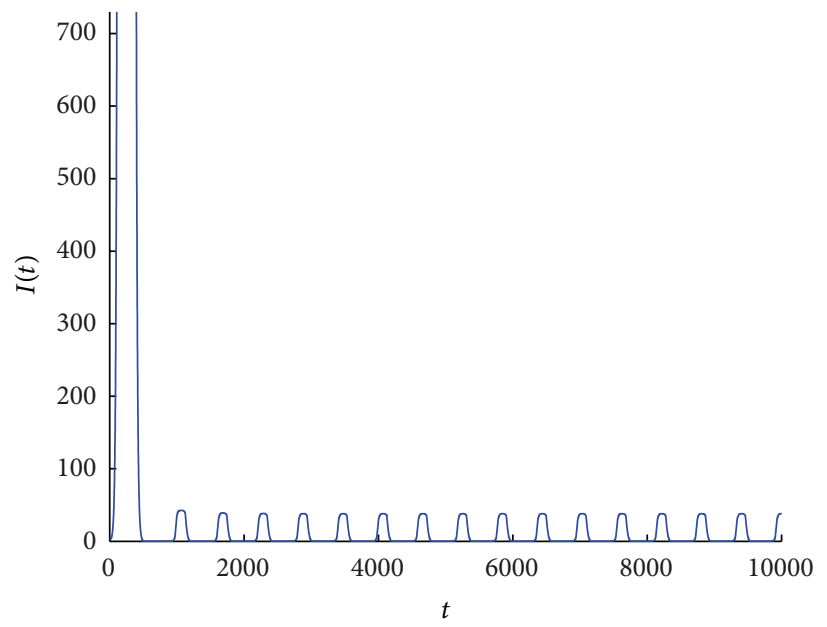

(b) Time series of $I(t)$ of the system

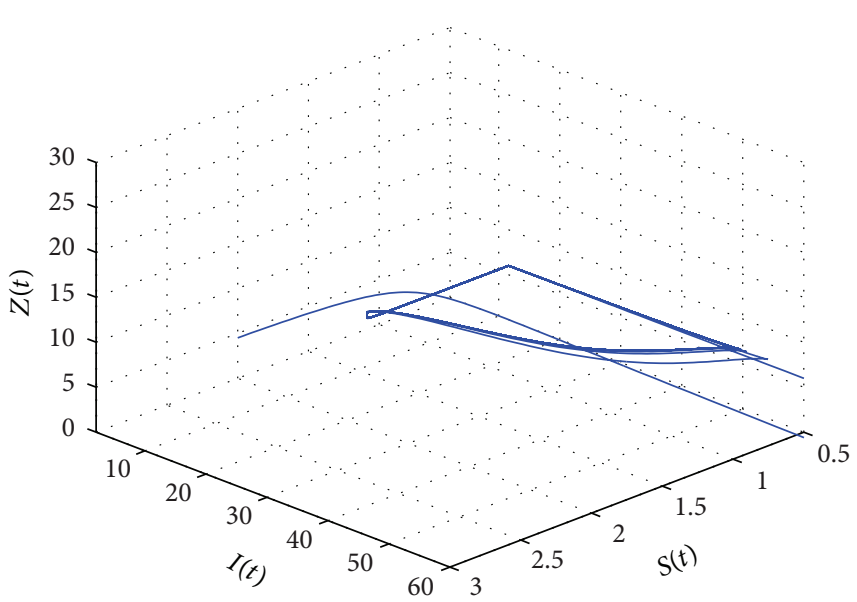

(d)

Figure 3: (a)-(d) showed that equilibrium $E^{*}$ of system (8) with initial condition $S(0)=2 ; I(0)=2 ; Z(0)=4.5 ; R_{0}=3.0303>1 ;$ and $\tau=2.56>\tau^{*}$ is unstable, that is, a periodic behavior at $\tau=2.56$.

$$
\begin{aligned}
\triangleq & \frac{i g_{20}}{\omega^{*} \tau^{*}} q(0) e^{i \omega^{*} \tau^{*} \theta}+\frac{i \bar{g}_{02}}{3 \omega^{*} \tau^{*}} \bar{q}(0) e^{-i \omega^{*} \tau^{*} \theta} \\
& +E_{1} e^{2 i \omega^{*} \tau^{*} \theta}
\end{aligned}
$$$$
W_{11}(\theta)=\frac{i g_{11}}{\omega^{*} \tau^{*}} \bar{q}(0) e^{-i \omega^{*} \tau^{*} \theta}+E_{2}
$$

Besides, $E_{1}, E_{2}$ are satisfied with the following equation:

$$
\begin{aligned}
& \left(\begin{array}{ccc}
2 i \omega^{*}-r\left(1-2 S^{*}\right)+G\left(I^{*}\right) & S^{*} G^{\prime}\left(I^{*}\right) & 0 \\
0 & 2 i \omega^{*}-S^{*} G^{\prime}\left(I^{*}\right)+\left(\mu_{1}+\gamma+\varepsilon\right)+\frac{b \omega}{\left(\omega+I^{*}\right)^{2}} & -G\left(I^{*}\right) \\
-\frac{1}{T} & 0 & 2 i \omega^{*}+\frac{1}{T}
\end{array}\right) E_{1} \\
& =2 \tau^{*} G^{\prime}\left(I^{*}\right) q_{1}(-1,1,0)^{T}+2 \tau^{*} \frac{b \omega}{\left(\omega+I^{*}\right)^{3}} q_{1}^{2}(0,1,0)^{T},
\end{aligned}
$$




$$
\begin{gathered}
\left(\begin{array}{ccc}
-r\left(1-2 S^{*}\right)+G\left(I^{*}\right) & S^{*} G^{\prime}\left(I^{*}\right) & 0 \\
0 & -S^{*} G^{\prime}\left(I^{*}\right)+\left(\mu_{1}+\gamma+\varepsilon\right)+\frac{b \omega}{\left(\omega+I^{*}\right)^{2}} & -G\left(I^{*}\right) \\
-\frac{1}{T} & 0 & \frac{1}{T}
\end{array}\right) E_{2} \\
=2 \tau^{*} G^{\prime}\left(I^{*}\right) q_{1}(-1,1,0)^{T}+2 \tau^{*} \frac{b \omega}{\left(\omega+I^{*}\right)^{3}} q_{1}^{2}(0,1,0)^{T} .
\end{gathered}
$$

Thus, we can determine $W_{20}(\theta)$ and $W_{11}(\theta)$ from (52). Furthermore, we can compute $g_{21}$ by (51). Thus we can compute the following values:

$$
\begin{gathered}
c_{1}(0)=\frac{i}{2 \omega^{*} \tau^{*}}\left(g_{20} g_{11}-2\left|g_{11}\right|^{2}-\frac{\left|g_{02}\right|}{3}\right)+\frac{g_{21}}{2}, \\
v_{2}=-\frac{\operatorname{Re}\left\{c_{1}(0)\right\}}{\operatorname{Re}\left\{d \lambda\left(\tau^{*}\right) / d \tau\right\}}, \\
\beta_{2}=2 \operatorname{Re}\left\{c_{1}(0)\right\}, \\
T_{2}=-\frac{\operatorname{Re}\left\{c_{1}(0)\right\}+v_{2} \operatorname{Re}\left\{d \lambda\left(\tau^{*}\right) / d \tau\right\}}{\omega^{*} \tau^{*}} .
\end{gathered}
$$

By the result of Hassard et al. [14], we have the following.

Theorem 7. In (54), the following results hold:

(i) the sign of $v_{2}$ determines the directions of the Hopf bifurcation: if $\nu_{2}>0\left(\nu_{2}<0\right)$, then the Hopf bifurcation is supercritical (subcritical) and the bifurcating periodic solutions exist for $\tau>\tau^{*}\left(\tau<\tau^{*}\right)$;

(ii) the sign of $\beta_{2}$ determines the stability of the bifurcating periodic solutions: the bifurcating periodic solutions are stable (unstable) if $\beta_{2}<0\left(\beta_{2}>0\right)$;

(iii) the sign of $T_{2}$ determines the period of the bifurcating periodic solutions: the period is increasing (decreasing) if $\beta_{2}>0\left(\beta_{2}<0\right)$.

\section{Numerical Simulations}

To demonstrate the theoretical results obtained from this paper, letting $G(I(t-\tau))=I(t-\tau) /(1+\alpha I(t-\tau))$, we will give some numerical simulations. We consider the hypothetical set of parameter values as follows.

(1) Consider $\mu_{1}=0.1 ; r=3 ; b=1 ; \gamma=0.05 ; T=4$; $\varepsilon=0.02 ; \alpha=0.2 ; \omega=0.4$. By directly computing, we obtain $R_{0}=0.1786<1$. According to Theorem 4, we know that the disease-free equilibrium of system (8) is locally asymptotically stable for this case (see Figures $1(\mathrm{a})-1(\mathrm{~d}))$

(2) Consider $\mu_{1}=0.01 ; r=3 ; b=0.1 ; \gamma=0.05 ; T=10$; $\varepsilon=0.02 ; \alpha=0.2 ; \omega=0.4 ; \tau=1.56$. By directly computing, we obtain $R_{0}=3.0303>1$. According to Theorem 4, we know that the disease-free equilibrium of system (8) is locally asymptotically stable for this case (see Figures 2(a)-2(d)).

(3) Consider $\mu_{1}=0.01 ; r=3 ; b=0.1 ; \gamma=0.05$; $T=10 ; \varepsilon=0.02 ; \alpha=0.2 ; \omega=0.4, \tau=2.56$. By directly computing, we obtain $R_{0}=3.0303>1$. According to Theorem 6, we know that the diseasefree equilibrium of system (8) is unstable for this case (see Figures 3(a)-3(d)).

\section{Conclusion}

In this paper, we formulate and analyze a new delayed epidemic model with information variable and limited medical resources, the conditions for Hopf bifurcation to occur are derived. By analyzing the model, we have found the existence of disease-free equilibria $E_{0}$ and $E_{1}$ and have a unique positive equilibrium $E^{*}$. The basic reproduction number $\mathscr{R}_{0}$ changes the stability of the disease-free equilibrium. When $\mathscr{R}_{0}<$ 1 , we discuss the stability of the disease-free equilibrium by analyzing the corresponding characteristic equations and constructing a Lyapunov functional, respectively. The conclusion reveals that the disease dies out and when $\mathscr{R}_{0}>1$, we also get the sufficient criteria of stability switch at the positive equilibrium. Using the time delay (i.e., incubation time) as a bifurcation parameter, the local stability of the endemic equilibrium is investigated, and the conditions for Hopf bifurcation to occur are derived. Using the normal form theory and the center manifold theorem introduced by Hassard et al., we have studied the direction and stability of the bifurcating periodic solutions. Our theoretical results show that the time delay $\tau$ must be responsible for the observed regular cycles of disease incidence.

Lastly, a numerical simulation provided that when $\mathscr{R}_{0}$ is less than 1 , the disease-free equilibrium is stable and while $\mathscr{R}_{0}$ is more than 1 , the disease-free equilibrium is unstable; that is, the endemic equilibrium exists (see Figure 1). Further, for $\tau>$ 0 , there will exist $\tau^{*} \in I$, such that the endemic equilibrium is asymptotically stable for $0<\tau<\tau^{*}$ (see Figure 2) and becomes unstable for $\tau$ staying in some right neighborhood of $\tau^{*}$, with a Hopf bifurcation occurring when $\tau=\tau^{*}$. If $\tau>\tau^{*}$, the endemic equilibrium is unstable (see Figure 3 ).

\section{Conflict of Interests}

The authors declare that there is no conflict of interests regarding the publication of this paper. 


\section{Acknowledgments}

The authors would like to thank the anonymous referees for their careful reading of the original paper and their many valuable comments and suggestions that greatly improved the presentation of this work. This work is supported by the Natural Science Foundation of Shanxi province (20130110022).

\section{References}

[1] J. W. Jia and Q. Y. Li, "Qualitative analysis of an SIR epidemic model with stage structure," Applied Mathematics and Computation, vol. 193, no. 1, pp. 106-115, 2007.

[2] J.-Q. Li, Z.-E. Ma, and J. Zhang, "Global analysis of some epidemic models with general contact rate and constant immigration," Applied Mathematics and Mechanics, vol. 25, no. 4, pp. 396-404, 2004.

[3] J. J. Wang, J. Z. Zhang, and Z. Jin, "Analysis of an SIR model with bilinear incidence rate," Nonlinear Analysis: Real World Applications, vol. 11, no. 4, pp. 2390-2402, 2010.

[4] G. Huang and Y. Takeuchi, "Global analysis on delay epidemiological dynamic models with nonlinear incidence," Journal of Mathematical Biology, vol. 63, no. 1, pp. 125-139, 2011.

[5] A. Korobeinikov, "Global properties of infectious disease models with nonlinear incidence," Bulletin of Mathematical Biology, vol. 69, no. 6, pp. 1871-1886, 2007.

[6] R. Xu and Z. E. Ma, "Global stability of a SIR epidemic model with nonlinear incidence rate and time delay," Nonlinear Analysis: Real World Applications, vol. 10, no. 5, pp. 3175-3189, 2009.

[7] Y. Enatsu, E. Messina, Y. Muroya, Y. Nakata, E. Russo, and A. Vecchio, "Stability analysis of delayed SIR epidemic models with a class of nonlinear incidence rates," Applied Mathematics and Computation, vol. 218, no. 9, pp. 5327-5336, 2012.

[8] A. d'Onofrio, P. Manfredi, and P. Manfredi, "Bifurcation thresholds in an SIR model with information-dependent vaccination," Mathematical Modelling of Natural Phenomena, vol. 2, no. 1, pp. 26-43, 2007.

[9] A. d'Onofrio, P. Manfredi, and E. Salinelli, "Vaccinating behaviour, information, and the dynamics of SIR vaccine preventable diseases," Theoretical Population Biology, vol. 71, no. 3, pp. 301-317, 2007.

[10] B. Buonomo, A. d'Onofrio, and D. Lacitignola, "Global stability of an SIR epidemic model with information dependent vaccination," Mathematical Biosciences, vol. 216, no. 1, pp. 9-16, 2008.

[11] E. Beretta and Y. Kuang, "Geometric stability switch criteria in delay differential systems with delay dependent parameters," SIAM Journal on Mathematical Analysis, vol. 33, no. 5, pp. 11441165, 2002.

[12] X. Y. Song, S. L. Wang, and J. Dong, "Stability properties and Hopf bifurcation of a delayed viral infection model with lytic immune response," Journal of Mathematical Analysis and Applications, vol. 373, no. 2, pp. 345-355, 2011.

[13] J. Hale and S. M. V. Lunel, Introduction to the Theory of Functional Differential Equations Methods and Applications, Spring, 1993.

[14] B. Hassard, N. Kazarinoff, and Y. Wan, Theory and Applications of Hopf Bifurcation, Cambridge University Press, Cambridge, UK, 1981. 


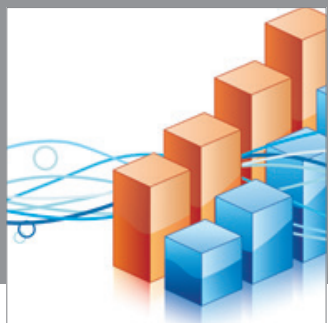

Advances in

Operations Research

mansans

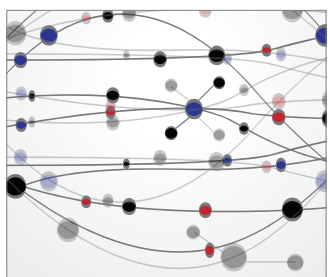

The Scientific World Journal
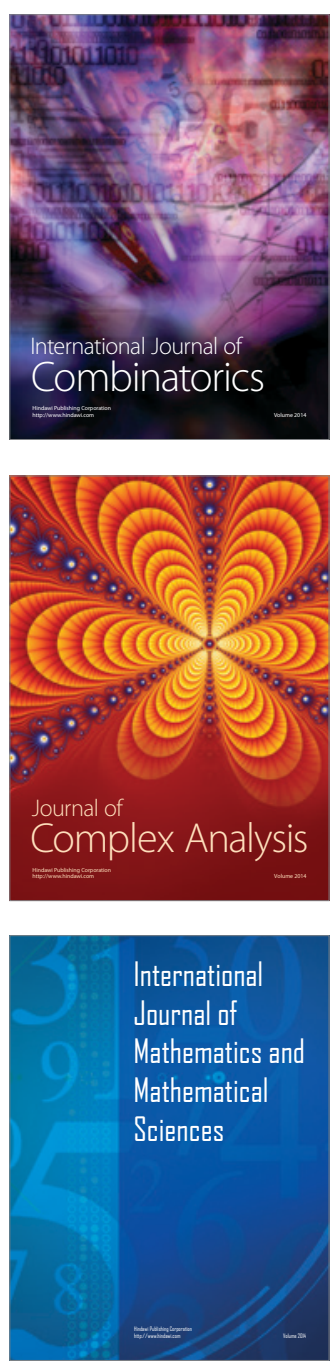
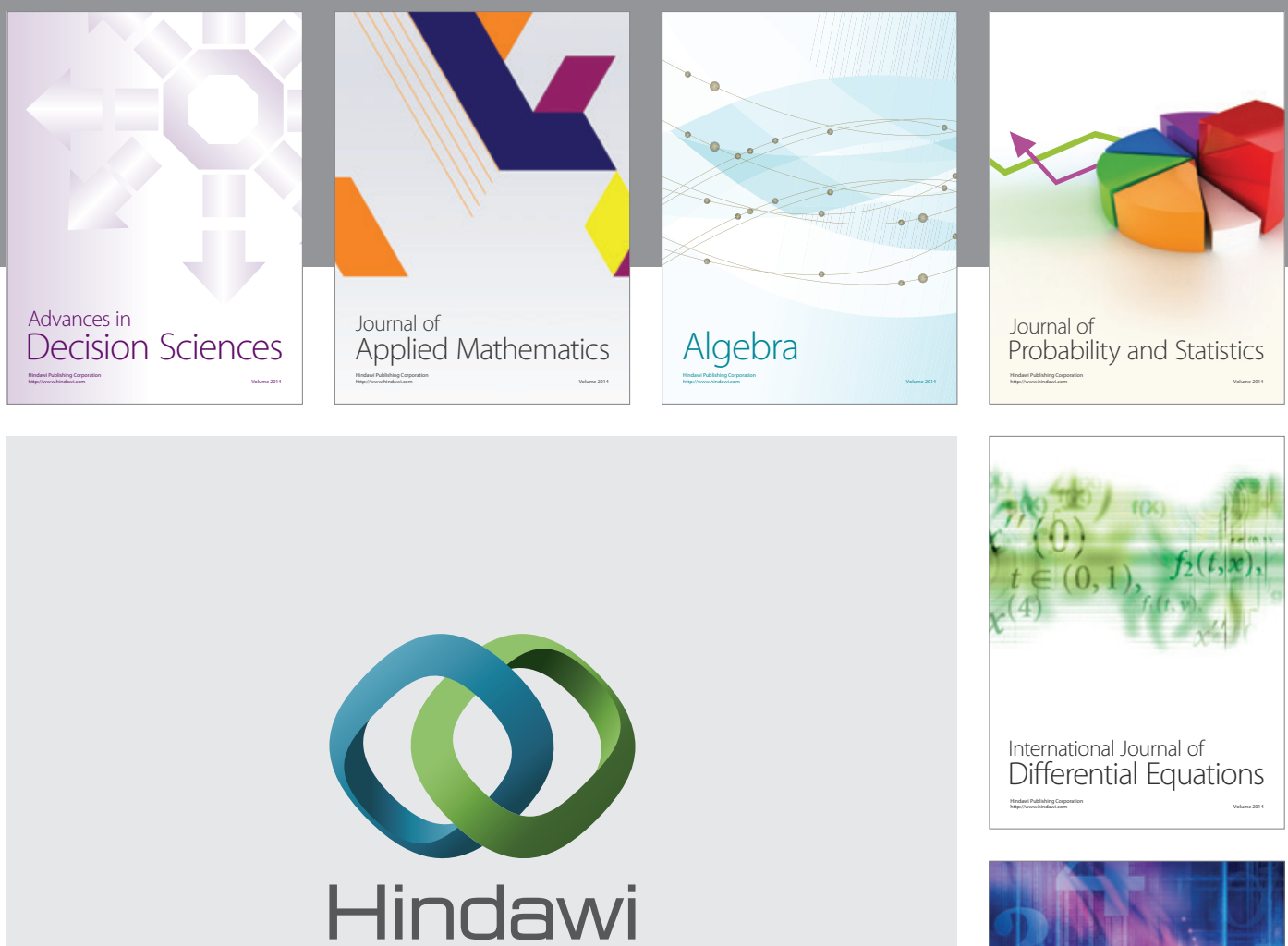

Submit your manuscripts at http://www.hindawi.com
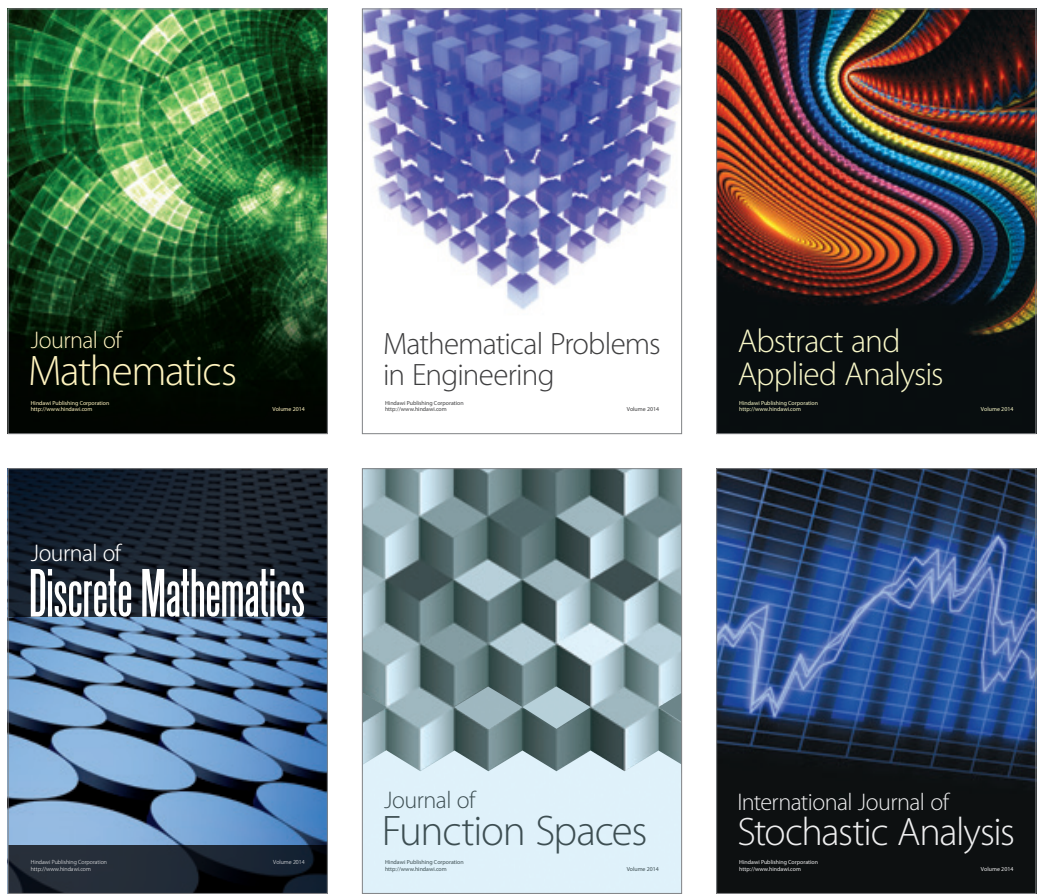

Journal of

Function Spaces

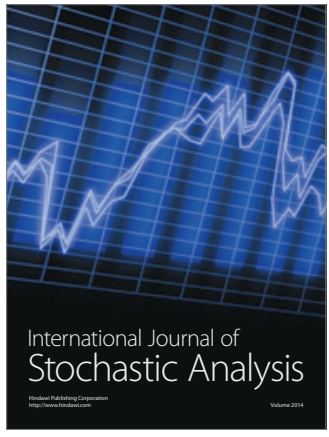

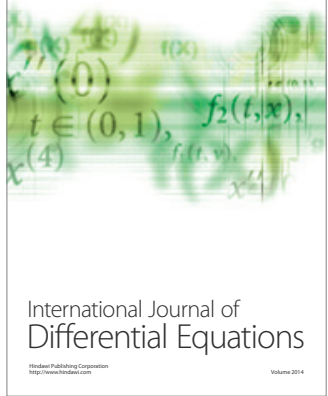
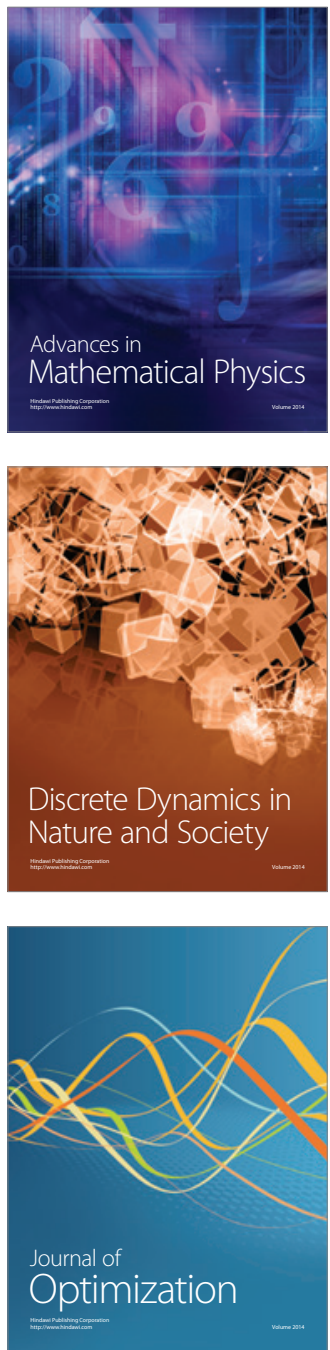